\title{
Added Value of In-Situ Methods in Usability Evaluation of a Self-Service Ticketing Machine with a View on Elderly Users: A Case Study
}

\author{
Elke E. Mattheiss ${ }^{1}$, Johann Schrammel ${ }^{1}$ and Manfred Tscheligi ${ }^{1,2}$, \\ ${ }^{1}$ CURE - Center for Usability Research and Engineering, Vienna, Austria \\ \{mattheiss, schrammel\}@cure.at \\ ${ }^{2}$ ICT\&S, University of Salzburg, Austria \\ manfred.tscheligi@sbg.ac.at
}

\begin{abstract}
Self-service systems are very common in the public space and usability plays a crucial role for user acceptance. We describe the evaluation of a self-service ticketing machine installed in trams in the city of Graz. Two insitu methods (behavior observation, contextual interview) and an online survey were used. We discuss advantages of the different methods experienced in the present study according to effort and output. Unsurprisingly online survey was advantageous in terms of time effort. Concerning output, behavior observation identified the most issues with specific interface objects of all methods. Furthermore we were able to identify usage information about the user group of elderlies. Online survey provided a higher number of general issues with the machine and recommendations for change than the two in-situ methods. Further results are discussed and limitations of the study are described in the paper.
\end{abstract}

Keywords: Usability evaluation, in-situ, behavior observation, contextual interview, online survey, self-service ticketing, case study, elderly users.

\section{Introduction}

Self-service systems are considered to be modern and efficient and are widely used in public transport. As they need to be operated by a broad range of users with heterogeneous technical abilities, the usability of these systems is essential.

There are various approaches how usability evaluation of computer systems can be performed. The Human-Computer Interaction (HCI) community has brought forth a large diversity of evaluation methods, whose suitability depends on the concrete situation of the evaluation including the computer system to evaluate, the context of use, and the kind of results striven for. Various classifications to categorize these methods according to key aspects can be found in previous literature, like user involvement (required, not required), context of evaluation (generic, application specific), type of results (qualitative, quantitative) [1], objective versus subjective measures, targeted usability aspect (effectiveness, efficiency, satisfaction) [2], study setting (laboratory, field), and target groups (e.g. elderly users [3]). In the present work we used two in-situ methods (behavior observation, contextual interview) and 
an online survey. The behavior observation consisted of twelve hours observing the buying process at the ticketing machine in the tram. For the contextual interview users were asked in the tram about issues with and attitudes towards the ticketing machine right after they used it. The online survey was retrievable for a week and the link was distributed by e-mail among acquaintances with prior experience with the ticketing machine. Therefore in-situ methods and online survey differed in the participants' context while they took part in the evaluation: in the context of system use (in the tram, nearby the ticketing machine, right after a buying process) and outside the context of system use (not in the tram, not right after a buying process). Another difference between the methods relate to the kind of information they are able to provide. Only behavior observation can provide performance data (number of specific kinds of errors in a given time period, duration of the buying process) but on the other side only contextual interview and online survey can assess the users' attitudes and opinions.

The focus of the present paper is on the advantages of each of the three used methods in terms of effort and output in the evaluation of a self-service ticketing machine. The evaluation was undertaken to find usability issues associated with the ticketing machine. However the focus of this case study is to report and reflect on the used methods in the particular context of a self-service ticketing machine and with a view on elderly users of the system. We describe the time effort for the evaluators and the participants in the different evaluation methods. Furthermore we consider the amount of different kinds of information (interface design issues, general issues with the machine, metric data, user ratings, and user recommendations) which the different methods are able to provide in our specific evaluation.

\section{Self-Service in Public Spaces}

Self-service machines are becoming wide-spread in the public space. Using selfservice machines is frequently more time and cost efficient than service by employees. Self-service technology has already transformed entire industries, and nowadays users are confronted with them at airport and travel kiosks, vending machines, food-ordering kiosks, self-checkouts, health care kiosks, and retail kiosks [4]. As self-service systems are intended to be handled without assistance, the usability of the systems plays an important role. [5] provides a review of design guidelines for public information kiosk systems dealing with recommendations for location, physical access, language selection, privacy, help, and input to the system.

Another crucial issue for the design of self-service systems is that they should be accessible for all kinds of users including those having physical or cognitive disabilities. Research efforts target this challenge by intelligent designs which are able to adapt their user interfaces to user characteristics like user heights, seeing ability, and motor abilities influencing the target hitting accuracy (e.g. [6]). Considering elderly people in the design of self-service system is crucial not only because they typically face a variety of impairments but also because age was found to have a negative influence on the preference for service technology over contact to personnel [7]. 
Researchers investigated the users' interaction with self-service systems in the public space. In [8] and [9] an extensive study about a ticketless travelling system in an airport train is presented. Using behavioral observations they found that users of the system did not actively request assistance and got quickly annoyed when they had problems to operate the machine [8]. That emphasizes the importance of designing easy to understand and operate systems. Usability is also one important factor contributing to the users' acceptance towards a system. According to the prominent Technology Acceptance Model [10] the perceived usefulness and the perceived ease of use are of primary relevance for the attitude towards using a system, which again is a factor for the actual system use. However, research work in the field of self-service ticketing systems (e.g. e-ticketing [11] and mobile ticketing [12]) claim that in addition to ease of use and usefulness also other factors like subjective norm, technology trust [11], social influence, cost, prior experience, risk, and use context [12] are important for the use intention. [13] found that the users' attitude towards self-service in railway ticketing and travel information was more positive when there was offered an interaction with an employee as a fall-back option.

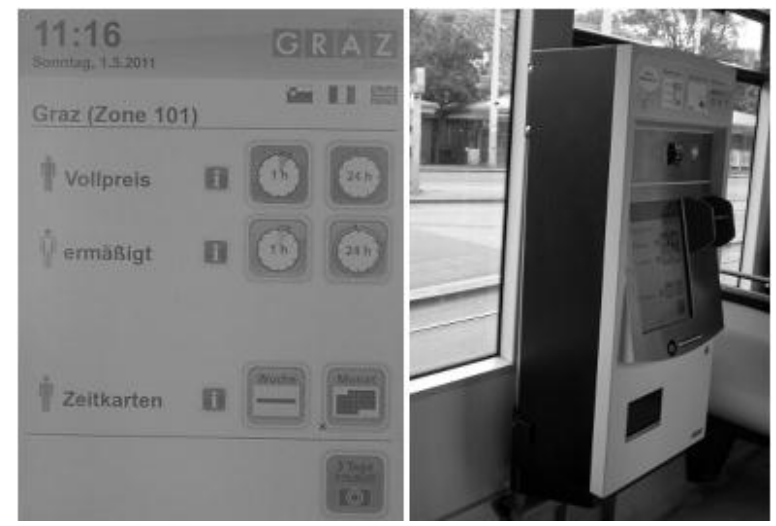

Fig. 1. User interface (left) and positioning (right) of the self-service ticketing machine.

In our case the ticket machines were installed in the trams, usually at the second entrance, oriented towards the left side of the tram (see Fig. 1). In this position the users' interaction with the ticketing machine is visible to bystanders. This is relevant for the user interaction because users may be embarrassed when struggling with the machine. As an alternative to using the machine, tickets are available in pre-sale. At the ticketing machine the users have the possibility to buy full fair and reduced tickets for one hour, 24 hours, 3 days (tourist ticket), a week, and a month (see Fig. 1 left). It is possible to pay with coins, credit card, or debit card. Since paying with card requires entering a PIN, trust and risk factors may be important for the user acceptance, because other passengers could potentially see the PIN. To enhance accessibility for a broader range of users the buying procedure is explained at the top of the machine, different languages can be selected, and the contrast settings can be changed. The specifics of the ticketing machine influenced the selection of appropriate evaluation methods, which are described in the next section. 


\section{Research Method}

For the evaluation of a self-service ticketing machine located in the trams not only the interface design, but also a lot of contextual aspects may contribute to the perceived usability of the system. Context is described in [14] as "...the sum of relevant factors that characterize the situation of a user and an application, where relevancy implies that these factors have significant impact on the user's experience when interacting with that application in that situation". In the present evaluation relevant context factors are for example sunlight towards the display, number of people in the tram, and movement of the tram. According to [15] a frequent reason for system failure is the fact that designers do not pay enough attention to the context of system use. Therfore we decided against conducting a traditional usability study in a laboratory setting, because the above stated context factors would be hard to simulate.

In-situ methods have the advantage to consider such context factors as they are conducted directly in the context of system use, and therefore overcome the problem of neglecting it. An example for an in-situ approach is ethnography which is roughly characterized by field work in a natural setting, providing a more complete context of activity [16], and typically consisting of a prolonged activity with a discursive and lengthy output [15] like rich descriptions of interactions. An obvious issue with this kind of traditional ethnography is the amount of time it requires. Therefore a "quick and dirty" ethnographic approach, with days of observation sparsely spread over a period of twelve months to evaluate a ticketless travelling system, was used in [8]. By these means it was possible to have a short and focused study. The authors gained with it a general understanding of the situation as well as a reality-check of the system in terms of an evaluation [9]. The authors present excerpts of their observations illustrating several problems and incidents as well as design implications.

Another cost efficient ethnographic approach named rapid ethnography is proposed by [17]. We considered some key aspects of this approach to develop our research method. The considered key aspects are to define specific questions (prior to the evaluation) that should be answered (to facilitate motivated looking and avoid a wide-angle lens approach), to choose a reasonable time sample (to ensure a representative and large sample of observations), and to use interactive research approaches like interviews (to understand the user behavior more rapidly in the field). We used the two methods behavior observation and contextual interviews as an approach of rapid ethnography. This combination of methods allowed us to gather behavioral data from the observations like performance data (e.g. task completion time, number of errors) as well as subjective attitudes and opinions of the users.

A survey is a completely different evaluation approach than ethnographic methods. A self-administered mail survey distributed to a random sample of 1000 users was used in [12]. Even less effort is needed when the survey is applied via an online survey tool and the link is distributed to acquaintances, asking them to forward the link to other potentially interested people. This approach was used by us for time and cost reasons. An obvious disadvantage of the survey approach is that it is only possible to gather subjective data about the users' attitudes without being able to capture time measurements and other performance data. 
The present study. In the following paragraphs the participants and the procedure of the conducted evaluation methods are described in detail. Behavior observation and contextual interview were conducted in-situ at the same time. The online survey was distributed a week after the in-situ evaluation was completed.

Setting of Behavior Observation and Contextual Interview. The behavior observations and the contextual interviews were conducted in the same time period by two evaluators in the trams near the ticketing machine. The study took place in June on a weekday and a weekend day at three different times of the day (about 8.00$10.00,12.00-14.00,16.00-18.00)$ to include a preferably broad range of different users regarding demographic characteristics (e.g. age) and travelling aim (e.g. way to work, travel during leisure time). Evaluators travelled for about two hours with a tramline from one final stop to the other.

Behavior Observation. The focus of the behavior observation was on issues with the user interface design and associated errors, but also other incidents were noted on a prepared protocol. Those incidents include duration of the buying process, type and amount of bought tickets, number of people waiting for buying a ticket, social interactions between users of the ticketing machine, sex of the users, and other user characteristics (e.g. children who are estimated by the observers to be aged under 14 years, elderlies estimated to be aged above 65 years, and physical limitations, foreign language as mother tongue) as effectively as possible. The evaluator observed the buying process of the users from behind. Participants of the behavior observation were all people who used the ticketing machine during the approximately twelve hour observation period. Altogether the buying processes of 122 (50 male, 72 female) users were observed, eight of them were classified according to their appearance as an elderly person older than 65 years. Table 1 lists characteristics of all the participants.

Contextual Interview. For the contextual interview the evaluator asked a user who just finished the buying process to answer some interview questions. Participants were chosen by chance and with a targeted rate of four interviews per hour. Alltogether 45 (25 male, 20 female) users with a mean age of 32 (standard deviation $(\mathrm{SD})=13.96)$ years were interviewed. One of them was aged above 65 years. The interview took about two-to-three minutes and included nine questions covering problems with the buying process, rating of the usability of the machine, rating of the design, frequency of using the ticketing machine, preference for buying the ticket from the tram driver instead of the machine, proposed changes, and accessibility for users with physical impairments. The answers of the interviewee were recorded with a dictation machine or noted down, if the user was not agreeable with the recording.

Table 1. Characteristics of the participants of the different methods.

\begin{tabular}{llll}
\hline & $\begin{array}{l}\text { Behavior } \\
\text { Observation }\end{array}$ & Contextual Interview & Online Survey \\
\hline Number & 122 & 45 & 26 \\
Sex & 50 male, 72 female & 25 male, 20 female & 10 male, 16 female \\
Mean Age & - & 32.00 years $\left(\mathrm{SD}^{*}=13.96\right)$ & 33.81 years $(\mathrm{SD}=13.00)$ \\
& & $\min =12, \max =73$ & $\begin{array}{l}\min =17, \max =70 \\
1\end{array}$ \\
$\begin{array}{l}\text { Elderlies } \\
(\mathbf{6 5}+)\end{array}$ & 8 & 1 & \\
*SD = Standard Deviation & &
\end{tabular}

*SD $=$ Standard Deviation 
Online Survey. The online survey was created with the open source survey application LimeSurvey [18]. The link to the survey was distributed in terms of a cost and time efficient approach to acquaintances of the authors' private and professional environment that were assumed to have prior experience with the ticketing machine in Graz. Moreover recipients of the link were asked to forward it to potentially interested people of their environment. By these means we aimed to spread the survey out to a more comprehensive sample. The survey took about ten minutes to be completed and covered in addition to the questions of the contextual interview also some questions related to demographic information and usage of the public transport system in general. The survey was online retrievable for one week and in this time period 26 participants completed it. This relatively small number of participants seems to be due to the instance that we announced the link by e-mail and in postings at social networking sites for only one time. Most participants completed the survey right after they received the link. Therefore if a larger sample is targeted the survey should be announced multiple times. In our case 26 participants (10 male, 16 female) with a mean age of $33.81(\mathrm{SD}=13.00)$ years sufficed. One participant aged above 65 took part in the survey.

\section{Costs and Benefits of the Used Methods}

In this part we present and discuss the used methods behavior observation, contextual interview, and online survey in terms of time effort (for evaluators and participants of the study) and output (number of identified usability issues, performance data, ratings, user recommendations). Furthermore we take a closer look on the data related to the elderly users. Because of the prevalence of qualitative data, the results remain mainly on a descriptive level.

\subsection{Time Effort}

When applying methods to evaluate a technology, there is always the question about which method suits best to obtain the highest output with the lowest effort. Reflecting on the methods used within the present evaluating study we collected the time effort we experienced in our specific evaluation. The extent of the applied methods are twelve hours behavior observation, 45 contextual interviews lasting two-to-three minutes (in our case accomplished in a twelve hour time period - but in principle also in less time possible), and 26 completed online surveys lasting about 10 minutes. Table 2 provides an overview about the time efforts for the three applied methods separated for the evaluation activities (preparation, execution for evaluator and participants, data entry, and data analysis). We classified the time effort into "none", "small", "medium", and "large". This classification is for comparing the time effort of the three methods in the different evaluation activities but not to compare the time effort between evaluation activities.

It is not surprising that overall the online survey has an advantage compared to the in-situ methods in terms of time effort for the evaluator. There is no time effort for the evaluator for execution and data entry, because this is accomplished automatically by 
the LimeSurvey application. The clear structure of the online form also facilitates the data analysis compared to the contextual interview, which is more opened and therefore often results in more chaotic data. Only the preparation time is higher than for the in-situ methods, because the online survey has - besides being planned content wise - also set up technically with the LimeSurvey application. For the participants filling out the online survey requires the greatest time effort.

We experienced the greatest time effort for the evaluator in the execution of the behavior observation. Of course the duration of observation was chosen to be twelve hours, but in the given context a significantly shorter observation period would have not been sufficient to gain an overview of the situation and observe enough buying processes to collect a reasonable number of usability issues.

Table 2: Overview of the time effort (classified into none, small, medium, and large) for the different methods experienced in the evaluation used.

\begin{tabular}{|c|c|c|c|}
\hline $\begin{array}{l}\text { Evaluation } \\
\text { Activity }\end{array}$ & $\begin{array}{l}\text { Behavior } \\
\text { Observation }\end{array}$ & $\begin{array}{l}\text { Contextual } \\
\text { Interview }\end{array}$ & $\begin{array}{l}\text { Online } \\
\text { Survey }\end{array}$ \\
\hline Preparation & Small & Small & Medium \\
\hline \multicolumn{4}{|l|}{ Execution } \\
\hline Evaluator & Large & Medium & None \\
\hline Participant & None & Small & Medium \\
\hline Data entry & Medium & Large & None \\
\hline Data analysis & Medium & Large & Medium \\
\hline
\end{tabular}

\subsection{Output}

The most crucial aspect of evaluation methods is the output they produce in terms of how many problems and recommendations for change are they able to identify. Since methods differ naturally in the kind of information they can provide (e.g. behavior observation cannot provide user ratings of usability) we reflect on the methods separately for different information types. For the analysis we collected all information gathered by the three evaluation methods in one list. Then we checked for each entry of the list from which methods they were identified. Finally we classified the information on the list according to their content into the five types: interface design issues, general issues with the ticketing machine, metric data, user ratings, and user recommendations.

Interface Design Issues. To find issues related to the interface design was the main focus of the evaluation and all three methods were potentially able to identify them. We only considered concrete issues with specific aspects of the design and neglected more general statements about the interface (e.g. "it's too complicated") for this analysis.

In sum 14 interface design issues were identified by all three methods together. The percentage of issues observed in the tram and the number of problems reported when the users were asked (in the tram or via online survey) are shown in Fig. 2 (three bars on the on the left side). The behavior observation found eight issues (57.14 
$\%$ ), the contextual interviews seven $(50.00 \%$ ), and the online survey only three (21.43\%). Having a look on the issues which were not identified by behavior observation, it becomes clear that the six remaining issues would be hard to identify by behavior observation, because they cannot be observed from the outside without knowing what the users' think. For example a user who has "problems reading the text in the given font size" looks for an observer from behind just like a user who has troubles with the interface, and would be noted as "being confused" but without having any concrete information about the problem. On the other hand behavior observation identified six issues which were not identified by the other two methods, which relate to specific interaction steps or objects. For example although we observed some users having problems with a button for adjusting the number of tickets to buy, none of the users reported a related issue when they were asked in the interview and online survey.

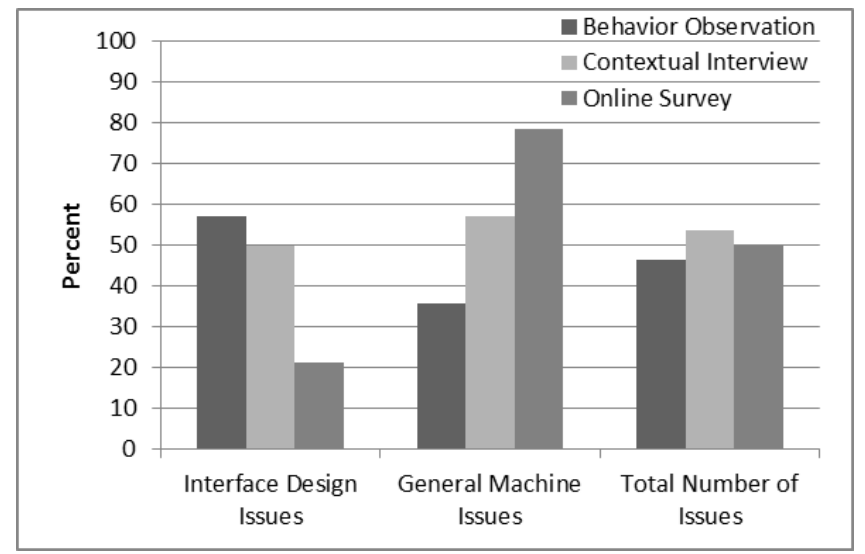

Fig. 2. Percentages of the identified interface design issues, general issues with the machine, and total number of issues (sum of interface design and general machine issues) for the different methods.

General Issues with the Machine. This category consists of all usability issues which were not related to the interface of the machine, but to the machine itself (e.g. size, positioning, functioning etc.).

In sum 14 general issues with the machine were identified by all methods together, five $(35.71 \%)$ by the behavior observation, eight $(57.14 \%)$ by the contextual interviews, and eleven $(78.57 \%$ ) by the online survey (see Fig. 2 three bars in the middle). The small number identified with behavior observation can be explained by the facts that some issues did not occur during the observation period (e.g. "machine did not work", "problem to get to the machine when it's crowded") and that some issues were not observable from the outside (e.g. "entering the PIN to pay with card feels unsecure when it's crowded"). Only one of the issues identified by behavior observation was not identified by the two other methods. It relates to a very specific functioning issue of the machine which occurred during the observation ("after 
canceling the payment a 30 seconds delay occurs until the home screen is displayed again") and which probably only few users experienced.

Other Data. The remaining information categories metric data, user ratings, and user recommendations are measurable with only a part of the three methods.

In the category metric data all information is collected which is able to answer questions about how often specific incidents happen or how long they take. Behavior observation is most suitable for this kind of information, because it provides a realistic and objective estimation about for example how often specific problems occur in a given time period, how often users ask for help, how many elderly people use the machine, how long the waiting queues are, and how long the buying process takes with cash or card payment (measured by the evaluator with a stopwatch).

User ratings can only be identified with the subjective methods (contextual interview and online survey). We asked our participants of the contextual interview as well as the online survey to rate the usability and the design of the user interface on a scale from one (best rating) to five (worst rating). To analyze potential differences in the ratings of the interview and the online survey data we used an unpaired twosample t-test. Looking at the usability ratings of our participants we found a significant difference $\left(\mathrm{t}_{69}=-3.12, \alpha<0.05\right)$ between the ratings in the contextual interview $($ mean $=1.78, \mathrm{SD}=0.64)$ and the online survey $($ mean $=2.35, \mathrm{SD}=0.89)$. The participants of the online survey rated the usability of the interface significantly worse than the participants of the contextual interview. No such difference was found for the rating of the design $\left(\mathrm{t}_{69}=-1.31, \alpha>0.05\right)$.

Like user ratings also user recommendations usually cannot be identified by behavior observation. In sum 34 user recommendations could be collected in the data of the contextual interviews and the online survey. The greater amount of concrete recommendations was provided by the participants of the online survey, who stated $24(70.59 \%)$. The participants of the contextual interviews provided $16(47.06 \%)$ recommendations. Interestingly only six issues were identified by both methods, which means that most of the issues were identified by just one of the methods. Having a closer look at the content of the recommendations reveals that $15(44.11 \%)$ recommendations are related to interface design (e.g. "place the buttons more apart from each other") and $19(55.88 \%)$ to general characteristics of the machine (e.g. "install the machines at the stations, not in the trams"). As described above we saw for the identified issues that online survey found considerably more general machine issues than the other two methods but less interface design issues. For the recommendations no such difference can be found, since 41.67 percent of the online survey participants' recommendations (and 37.50 percent of the contextual interview participants') are related to interface design.

\subsection{View on Elderly Users and Accessibility}

In this section we take a closer look on the elderly users and accessibility aspects evaluated. Due to the limited number of elderly participants we will not reflect on the differences between methods, but highlight findings approachable only by specific methods. 
As stated above only it is only assessable by behavior observation how often specific incidents happen. A first result in regard to elderly users is the surprisingly small number of elderlies using the ticketing machine - only 8 of 122 observed users $(9.76 \%)$ were classified as elderlies. Another interesting finding of behavior observation is that six of the eight as elderly users had problems with the buying process $(75 \%)$, but only 23 of the 114 other users $(20.18 \%)$. Furthermore three of the four users $(75 \%)$ asking other passengers for help were elderly users. These results point out the difficulties elderly users have with self-service technology very clearly. Another finding of the behavior observation relates to the accessibility features of the machine (changing of language and contrast). In the whole observation period none of the users changed the contrast to the mode which is easier to read for visually impaired. Furthermore only one user changed the language settings, although there were several users observed who had problems with the German language (which became obvious when they were asked for an interview).

\section{Conclusions}

In the present paper we presented and reflected on three different methods (behavior observation, contextual interview, online survey) used in the evaluation of a self-service ticketing machine in the public transport system of Graz, Austria. The main focus of the evaluation was to identify usability issues with the ticketing machine. Altogether 28 issues (interface design issues and general issues with the machine) were identified, and each method could identify about 50 percent of the issues (see also Fig. 2 three bars on the right side). A closer look on the different types of information reveals some interesting results, which are described in the following paragraphs.

The in-situ methods behavior observation and contextual interview identified a higher number of concrete interface design issues than the online survey. Especially behavior observation provides valuable information about when and where problems occur in the interface interaction process. When asking users' directly to describe the problems they have with an interface, they are often not more concrete than "it is too complicated" - probably because they are unconscious about the origin of the problem. Another advantage of behavior observation is that it can provide information and metrics about how often problems really occur in a given period of time. This cannot be provided by subjective data and can help not to overestimate the severity of an issue raised by user statements. Furthermore it can tell us something about the behavior of specific user groups like how many elderly users are using the machine and face problems. However, a big disadvantage of behavior observation, which is also reflected in our data, is that it can only capture what is actually observable "from the outside" and happens during the observation period. For a clearer understanding of the users' reasons and difficulties as well as to include also incidents apart the observation period, objective behavior observation should be supplemented with a subjective method.

With subjective methods - in contrast to behavior observation - also user ratings and user recommendations are recognizable. Comparing the usability ratings of the 
participants of contextual interview and online survey shows a significant difference. One possible explanation for that difference is that participants tend to be more critical in an anonymous online survey than in a one-on-one interview.

The online survey found less interface design issues but more general issues with the machine than the behavior observation and the contextual interview. This may indicate that general issues with the ticketing machine are very prominent in the participants' memories, but that interface design issues require some kind of proximity to the subject of interest. However when it comes to recommendations, the online-survey participants target the interface design almost equally often like general characteristics of the machine. Furthermore the participants of the online survey provided the highest number of recommendations for change. The absence of time pressure and the possibility to think carefully about the recommendations may be a factor for that instance.

\section{Limitations and Outlook}

With the present paper we aim to reflect on our experiences with different common methods (behavior observation, contextual interview, online survey) in the field of self-service evaluation. However, the presented results have some limitations. The most important limitation relates to the two incomparable samples of the in-situ method and the online survey due to the different recruiting approach of participants. For the in-situ methods all kinds of users of the ticketing machine were asked by chance to be part of an interview, but the online survey was distributed by e-mail and social network sites only to a selective sample of computer and internet users. Therefore besides the different sample size, we have to assume also differences in user characteristics like technophilia. Technophile people may provide more concrete feedback concerning usability issues due to their advanced experience with technology. Furthermore we cannot assume the same degree of heterogeneity in the online-survey-sample than in the in-situ-sample. Another limitation concerns the data analysis. We only considered the number of issues and recommendations identified by the different methods, but not the quality. Involving the usefulness of user statements somehow would be an interesting area of further research. Another area for future investigation could include different user groups systematically in the study design. It would be interesting to investigate if the results of the methods differ between elderly and other users. A last limitation concerns the interpretation of the presented results in terms of a case study, which means that they apply to the present evaluation conducted in the specific context of self-service ticketing. It is conceivable that the comparison between the methods will lead to other results if the context or concrete procedure changes. Nevertheless it provides valuable information for evaluators and a basis for further investigations in regard to added value of in-situ methods. 
Acknowledgments. The research presented is conducted within the Austrian project "AIR - Advanced Interface Research" funded by the Austrian Research Promotion Agency (FFG), the ZIT Center for Innovation and Technology and the province of Salzburg under contract number 825345 . We would like to thank Holding Graz Linien for access and assistance in doing the fieldwork.

\section{References}

1. Bowman, D., Gabbard, J.L., Hix, D.: A Survey of Usability Evaluation in Virtual Environments: Classification and Comparison of Methods. In: Presence: Teleoperators and Virtual Environments, 11(4), 435--455 (2002)

2. Hornbæk, K.: Current Practice in Measuring Usability: Challenges to Usability Studies and Research. International Journal of Human-Computer Studies, 64(2), 79--102 (2006)

3. Holzinger, A., Searle, G., Kleinberger, T., Seffah, A., Javahery, H.: Investigating Usability Metrics for the Design and Development of Applications for the Elderly. In K. Miesenberger et al. (eds.) ICCHP 2008, LNCS 5105, 98--105 (2008)

4. Castro, D., Atkinson, R., \& Ezell, S. (2010). Embracing the self-service economy. The Information Technology \& Innovation Foundation.

5. Maguire M.C.: A Review of User-Interface Design Guidelines for Public Information Kiosk Systems. Int J Hum-Comput Stud, 50, $263--286$ (1999)

6. Hagen, S., Sandnes, F.E.: Toward Accessible Self-Service Kiosks through Intelligent User Interfaces. Pers Ubiquit Comput, 14, 715--721 (2010)

7. Simon, F., Usunier, J.-C.: Cognitive, Demographic, and Situational Determinants of Service Customer Preference for Personnel-in-Contact over Self-Service Technology. International Journal of Research in Marketing, 24(2), 163--173 (2007)

8. Kristoffersen, S., Bratteberg, I.: Design Ideas for IT in Public Spaces. Pers Ubiquit Comput, 14, 271--286 (2010)

9. Bratteberg, I., Kristoffersen, S.: Irreversibility and Forceback in Public Interfaces. Proceedings of NordiCHI'08, 63--72 (2008)

10. Davis, F., Bagozzi, R., Warshaw, P.: User Acceptance of Computer Technology: A Comparison of Two Theoretical Models. Management Science, 35(8), 982--1003 (1989)

11. Lee, C.B.P., Wan, G.: Including Subjective Norm and Technology Trust in the Technology Acceptance Model: A Case of E-Ticketing in China. The DATA BASE for Advances in Information Systems, 41(4), 40--51 (2010)

12. Mallat, N., Rossi, M., Tuunainen, V.K., Öörni, A.: An Empirical Investigation of Mobile Ticketing Service Adoption in Public Transport. Pers Ubiquit Comput, 12, 57--65 (2008)

13. Reinders, M.J., Dabholkar, P.A., Frambach, R.T.: Consequences of Forcing Consumers to Use Technology-Based Self-Service. Journal of Service Research, 11, 107--123 (2008)

14. Jensen, K.L.: RECON - Capturing Mobile and Ubiquitous Interaction in Real Contexts. In: Proceedings of MobileHCI'09 (2009)

15. Hughes, J., King, V., Rodden, T., Andersen, H.: Moving out from the control room: ethnography in system design. In: Proceedings of the Conference on Computer supported cooperative work, 429--439 (1994)

16. Blomberg, J., Giacomi, J., Mosher, A., Swenton-Wall, P.: Ethnographic Field Methods and Their Relation to Design. In: Dchuler, C., Namioka, A. (eds.) Participatory Design: Principles and Practices, pp. 123--156. Erlbaum, Hillsdale, N.J. (1993)

17. Millen, D.R.: Rapid Ethnography: Time Deepening Strategies for HCI Field Research. In: Proceedings on Designing Interactive Systems: Processes, Practices, Methods, and Techniques, 280--286 (2000)

18. LimeSurvey open source survey application, http://www.limesurvey.org/ 